Research Article

\title{
Evaluation of the Linear Dimensional Changes and Hardness of Gypsum Product / Stone Type IV after Adding Silica Nanoparticles
}

\author{
Ola Mohammed Aljubori, Ali Mohammad Ali Aljafery , Raja'a Mahdi Al-Mussawi \\ Department of Prosthodontics, College of Dentistry, University of Kufa, Najaf, Iraq. \\ —Corresponding authors. E-mail: alim.aljaafri@uokufa.edu.iq, tel. 009647831520636
}

Received: Mar. I, 2020; Accepted: Jul. 3, 2020; Published: Aug. II, 2020

Citation: Ola Mohammed Aljubori, Ali Mohammad Ali Aljafery, and Raja'a Mahdi Al-Mussawi, Evaluation of the Linear Dimensional Changes and Hardness of Gypsum Product / Stone Type IV after Adding Silica Nanoparticles. Nano Biomed. Eng., 2020, 12(3): $227-23$ I.

DOI: 10.5101/nbe.v12i3.p227-231.

\begin{abstract}
Dental gypsum is routinely used as an investing and molding material for prosthodontics restoration with some acceptable linear dimensional changes in the finished restorations. This study aimed to evaluate linear dimensional stability and surface hardness of dental stone type IV when adding silica nanoparticles to it. 40 type IV stone specimens were prepared from stainless steel molds for linear dimensional stability and plastic mold for hardness, 20 of which contained silica nanoparticles (test group) while the other 20 were without any addition (control group). Each group was divided into subgroups containing 10 specimens for each one in order to compare the dimensional stability and hardness of them. The linear dimensional changes were measured using a digital caliper, while the hardness was tested with Vickers' hardness test. The data were analyzed with t-test (two-sample assuming unequal variances) and p-value. The mean value of linear dimensional changes for the control group was -0.396 and -0.386 for specimens of the test group. Mean hardness was 50.638 for the control group and 53.295 for the test group. The difference was significant for linear dimensional changes and highly significant for hardness. Adding of silica nanoparticles improved the hardness and reduced the linear dimensional changes of type IV stone.
\end{abstract}

Keywords: Gypsum product; Type IV stone; Dimensional stability; Surface hardness; Silica nanoparticles

\section{Introduction}

Dental casting is an important step during the process of dental prostheses. The progress in both materials and techniques directly enhance impressions and working casts that are crucial for the success of clinical work [1].

For decades, gypsum products have been used in dentistry to generate study and master casts, on which dental appliances are make-up [2]. Gypsum products are classified according to their particle size, as well as their properties depending on particle size [3]. According to the International Standards Organization, type IV dental stone are commonly used for constructing working casts on which fixed and removable restoration are constructing. It is characterized according to economy, easy to handle and manipulate, with superior compressive strength, hardness and dimensional stability [4-6].

The stone die must be hard enough to resist abrasion 
or deformation caused by scratching force applied on their surface [4, 7]. For complicated prosthodontics procedures, dental stones have low desired strength, low reproduction of the fine details and low abrasion resistance [8].

In general, gypsum products are commonly used material because their properties can be altered by physical and chemical methods [9]. To improve the strength of dental materials, inorganic filler particles are applied [10]. There are different types of inorganic filler particles such as quartz, colloidal silica, and silica glass containing barium, strontium, and zirconia. Also, the properties of the materials can be affected by the shapes and sizes of filler particles [11, 12]. Nowadays, the application of nanotechnology is considered an important and recent change in inorganic filler that is important to the progress of dental materials and improving their mechanical properties [9].

Nanotechnology is widely used in dentistry and many studies were done to investigate its anticipated gains. It is important to know how to use this technology and get benefits of it [13].

Nanomaterials are used with ceramic, metal, resin [14] and composite material [15] to improve their properties such as flexibility, strength, plasticity, mechanical compatibility and biocompatibility, as well as to reduce the porosity in the materials and make the modulus of elasticity similar to the form of natural bone [14].

Recently, many investigators have studied the effect of adding different types of functionalize nanoparticles to PMMA and evaluated the effect of these particles on PMMA properties after adding different shapes, sizes, and, ratios of them [13].

These nanoparticles have a small size, large surface area and high interactions between its interface and polymer matrix. So they can promote the physical properties of the polymer matrix $[15,16]$.

New investigations are anticipated with the aiding of this technology regarding the use of unfunctionalized nanoparticles and their effect on the mechanical properties of dental stone because there hasn't been sufficient research done yet.

The aim of this study was to evaluate and compare the linear dimensional changes and Vickers hardness of type IV dental stone materials with the same stone after adding silica nanoparticles. The null hypothesis was that nanoparticles would not adversely affect their properties.

\section{Experimental}

Two groups were prepared from 40 stone specimens (i.e. each group contains 20 specimens). The control group was made of 10 specimens prepared without silica nanoparticles, while the comparative group was consisted of 10 specimens that were prepared by adding silica nanoparticles.

\section{Linear dimensional changes Specimens' preparation}

For the evaluation of linear dimensional changes, a stainless steel mold was prepared with a $30 \mathrm{~mm}$ diameter and a $3 \mathrm{~mm}$ height from its top part. This top was supported as single structure by base 38 $\mathrm{mm}$ diameter and $30 \mathrm{~mm}$ in height. To make this mold with a shoulder suitable for a circular moving frame, the height of $6 \mathrm{~mm}$ permitted us to make a sample of $3 \mathrm{~mm}$ in thickness after taking a print of the upper surface (Fig. 1). The top surface of this mold contained 2 lines with triangular cross-sections and a $90^{\circ}$ base angle and $75 \mu \mathrm{m}$ width. These 2 lines were separated by a distance of $25 \mathrm{~mm}$. Based on the recommendations of ISO 4823: 1992 test method 7.7, the impression was mixed according to manufacture

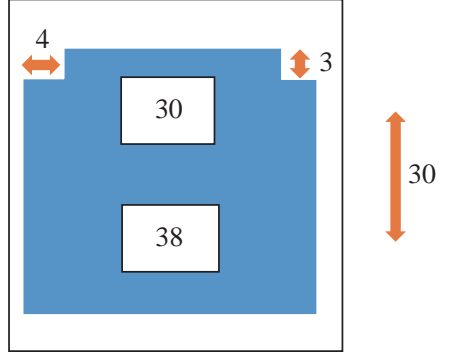

(a)

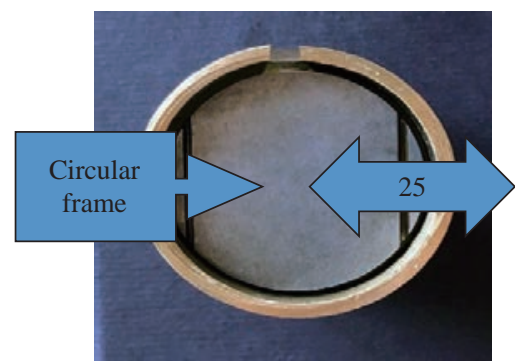

(b)

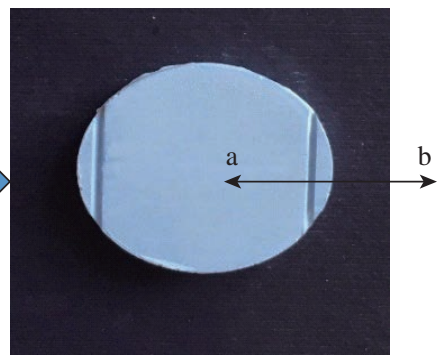

(c)

Fig. 1 (a) Diagram illustrating a stainless steel mold; (b) stainless steel mold with a circular moving frame rest on base shoulder; (c) stone specimens. 
instruction at room temperature $23^{\circ} \mathrm{C}$ and added with silicone impression material (Zhermack, zetaplus soft / putty, easy mix / Italy), which was poured with type IV stone (Elite Model, Zhermach, Italy). The mixing of stone was carried out according to the manufacturing instructions:, water / powder ratio $21 \mathrm{~mL} / 100 \mathrm{~g}$ at the same room temperature using a special mixer with vibration to decrease air bubbles (for the control group), while for the comparative group, $2 \%$ silica nanoparticles (Sigma-Aldrich, Germany) with 60-70 $\mu \mathrm{m}$ in size were mixed in previous manner. But the weight of stone powder decreased $2 \%$ for the weight of nanoparticles: water / powder ratio $21 \mathrm{~mL} /(98 \mathrm{~g}$ stone +2 g nano powder). To obtain a uniform blend and identical distribution of silica nanoparticles, ultrasonic mixer and high-energy ball milling were used, ball milling showed the best results, and then the manufacturing instructions were followed in mixing with water which was measured for mixing with stone powder.

\section{Measurements}

Digital microscope (Dino-lite $\times 200$ magnification, Taiwan) was used to evaluate the linear dimensional precision of stone specimens and to compare them with that of a stainless steel mold. All specimens were examined by measuring the straight-line distance between the 2 cross lines, and the measurements were recorded to the nearest $0.005 \mathrm{~mm}$. The measurements were carried out at $\times 90$ magnification under reproducible low-angle lighting. Then, the findings of the dimensional accuracy were reported as the changes took place in linear dimensions (AL). The following equation was used to determine AL:

$\mathrm{AL}=100$ (L2 - L1), where L1 was the span between 2 dotes (a and b) measured on the stainless steel mold, and L2 was the same span (a to b) measured on the gypsum model (according to the ISO specification number 4823 recommendation).

\section{Hardness \\ Specimen's preparation}

A plastic mold with dimensions of $10 \mathrm{~mm} \times 10$ $\mathrm{mm} \times 2 \mathrm{~mm}$ (Fig. 2) was prepared for evaluating the hardness test. A special mold container was used to take impressions with adding silicon impression material (Zhermack, zetaplus soft/putty, easy mix/ Italy) and poured to obtain 20 dies, 10 of which were poured with type IV stone (Elite Model, Zhermach, Italy) contained silica nanoparticles while the other

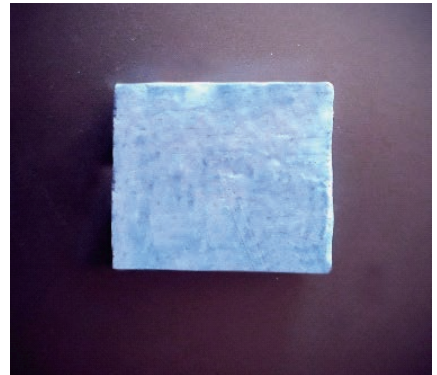

Fig. 2 Stone sample for hardness.

10 were poured with type IV stone without adding silica nanoparticles in a same manner described in Specimens> preparation for linear dimensional changes.

\section{Measurements}

Vickers' hardness test was used because of its exceptional capability to differentiate among solid substances having high rigidity [17]. This test was performed using a Micro-Hardness Vickers tester device (Zweick, Germany). The micro hardness device was supplied with a moving stage which had an accuracy of about $0.01 \mathrm{~mm}$, an eyepiece with a magnification of $\times 100$ and caliper for measuring. This provided the device with a resolution for measuring

Bar chart of linear dimensinal change

(b)
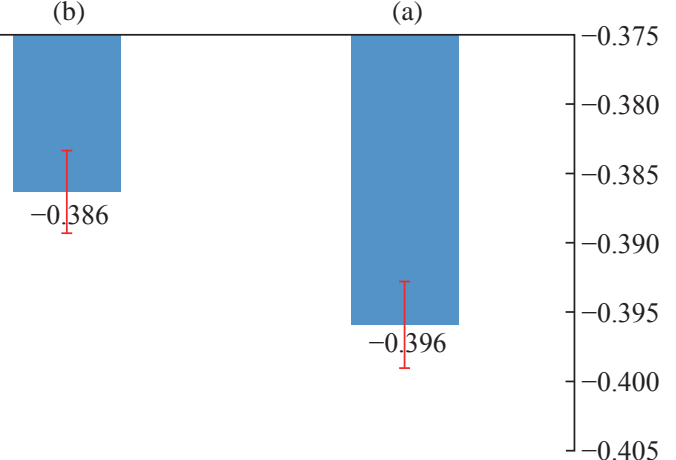

Fig. 3 The distribution of mean values for linear dimensional changes with standard error: (a) Control group; (b) comparative group.

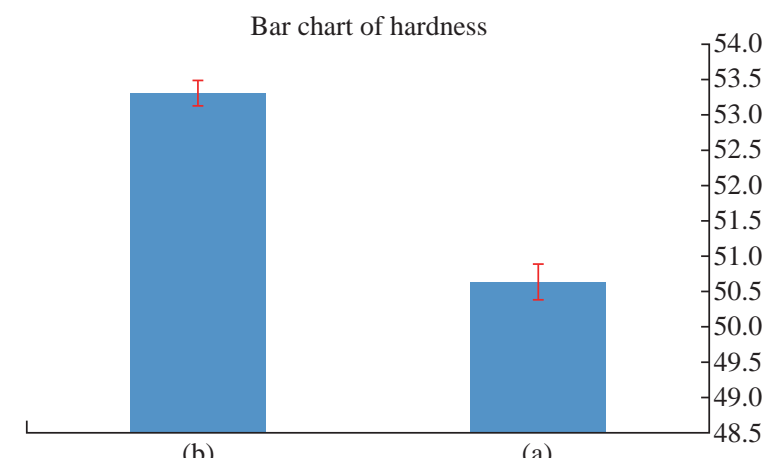

(b)

(a)

Fig. 4 The distribution of mean values for hardness with standard error: (a) Control group; (b) comparative group. 
reaches to $0.001 \mathrm{~mm}$.

The diagonal distance of the indentation mark was measured for each stone die specimen in the identified 3 marks. The mean value for each specimen was calculated. Then, the actual Vickers hardness value for each stone die specimen was calculated using the following equation:

$\mathrm{HV}=\mathrm{W} \times \mathrm{k} / \mathrm{d}^{2}$, where the weight $(\mathrm{w})$ was 50 grams, $\mathrm{k}$ was a constant for the diamond indentation angle, and d was the diagonal distance of the indentation mark. The data were analyzed with t-test (two-sample assuming unequal variances) and p-value.

\section{Results and Discussion}

Mean, standard deviation and standard error regarding the linear dimensional changes are presented in Table 1.

The independent samples' t-test showed a significant difference between tested groups regarding linear dimensional changes $(p \text {-value }=0.0377)^{*}$.

Mean, standard deviation and standard error regarding the hardness are presented in Table 2.

The independent samples t-test showed a highly significant difference between tested groups regarding hardness $(\mathrm{p} \text {-value }=0.00)^{*}$.

Usually, the casts and die materials must be characterized as dimensional stability, ability to reproduce fine details, solidified to a very hard and strong mass for abrasion resistance, easy for manipulation, no reaction with the impression material, have adequate transverse strength to withstand the force applied to it [1]. Frequently, dental stone is used to fabricate master casts [5] because it is economical, easy to handle and available in the market and compatible with impression material [8] with some limitations like' weak breakage resistance and dimensional inaccuracy [1].

The hardness and dimensional stability are important factors for successful dental restoration because they directly affect the accuracy, friction resistance and marginal fit of the restorations [1].

Adding the inorganic filler (silica nanoparticles) to the dental stones enhanced the mechanical properties of them. This filler was selected due to the successful results obtained when it was utilized for many experimental and commercial dental materials [9].

The current study was carried out by adding pure silica nanoparticles without functionalization to dental stones. The addition of silica nanoparticles leads to decreasing the linear dimensional changes of type IV dental stone and increasing their hardness significantly. This result can be attributed to that silica nanoparticles can enter the internal space between dental stone particles and occupy the present voids releasing air bubbles. There is no chemical reaction or bonding occurs between non-functionalized silica nanoparticles and stone / water mixture. However, the results may be justified that the small nanoparticles size and their large surface area lead to reducing surface tension area and increasing the wettability of hemihydrate (dental stone) to the water. The rate of solubility of the hemihydrate will be increased, and therefore a more rapid rate of crystallization will occur.

Consequently, the porosity of dental stone decreases, leading to restricting the linear dimensional changes and cracking propagation in dental model, which clearly improves the linear dimensional changes and the hardness.

These findings confirmed the results obtained by De Cesero et al. which showed improved surface roughness values of the stones after adding silica nanoparticles. Functionalizing the stone / water

Table 1 Mean linear dimensional changes, SD and SE of stone with and without adding silica nanoparticles

\begin{tabular}{ccc}
\hline Materials & Mean & Standard deviation \\
\hline Linear dimensional changes without adding silica nanoparticles & -0.396 & 0.0084 \\
Linear dimensional changes with silica nanoparticles & -0.386 & 0.0026 \\
\hline
\end{tabular}

Table 2 The mean hardness, SD and SE of stone with and without adding silica nanoparticles

\begin{tabular}{cccc}
\hline Materials & Mean & Standard deviation & Standard error \\
\hline Hardness without adding silica nanoparticles & 50.638 & 0.79 & 0.25 \\
Hardness with silica nanoparticles & 53.295 & 0.56 & 0.17 \\
\hline
\end{tabular}


mixture by silica nanoparticles led to deposition of these nanoparticles between the stone particles in the spaces which were occupied by the adsorbed water. The deposition occurred because of the functionalized nanoparticles, which was able to bind to the water molecules in the powder / liquid mixture. Thus, the surfaces of the stones became smoother, with smaller inter particle spaces [9].

In study of Vozza et al.[18], they evaluated the dimensional stability of silica reinforced polyurethane for casts, demonstrating the dimensional stability of silica reinforced polyurethane resin was higher than in 2 synthetic type IV and type V plasters.

Accoding to the data obtained from Junior et al. [1] who analyzed linear dimensional change of different materials used for casting dental models, the plasters were superior in reproducing the actual measurements of the original matrix than resin materials.

\section{Conclusions}

Within the limitations of the study, the following conclusion may be drawn: The surface hardness of dental stone was highly significantly increased when adding non-functionalized silica nanoparticles; the linear dimensional changes were significantly decreased when adding non-functionalized silica nanoparticles.

\section{Conflict of Interests}

The authors declare that they have no conflict of interest.

\section{References}

[1] E. Vieira, D. Silva, G.A.P. De, et al., Analysis of linear dimensional change of different materials used for casting dental models: plaster type 4, nanocomposites carbon nanostructures, polyurethane resin and epoxy resin. $J$ Dent Health Oral Disord Ther, 2018, 9(2): 200-205.

[2] K.J. Anusavice, R.W. Phillips, C. Shen, et al., Philip's science of dental materials, $12^{\text {th }}$ ed. Elsevier/ Saunders, 2013: 588.

[3] S.S. Kumararama, R. Chowdhary, Evaluation of linear dimensional changes of investment material when various combinations of dental plaster and dental stone are used as an investment material. IJPRD, 2016, 6(1): 1-5.

[4] M. Aurélio, Linear dimensional change, compressive strength and detail reproduction in type IV dental stone dried at room temperature and in a microwave oven. $J$ Appl Oral Sci, 2012: 588-593.

[5] B.J. Kenyon, M.S. Hagge, C. Leknius, et al., Dimensional accuracy of 7 die materials. J Prosthodonics, 2005, 14(1): 25-31.

[6] P. Duke, Study of the physical properties of type IV gypsum, resin-containing, and epoxy die materials. $J$ Prosthet Dent, 2000, 83(4): 466-473.

[7] V. Narayanan, J. Padmanabhan, In vitro comparison of dimensional stability of stone dies obtained from two elastomers. International Journal of Prosthodontics and Rstorative Dentistry, 2011, 1(13): 169-173.

[8] G.R.R. Lillywhite, F. Vohra, Influence of polyurethane resin dies on the fit and adaptation of full veneer crowns. Indian Journal of Dental Research, 2015, 26(1).

[9] L. De Cesero, M. Nunes, The addition of silica nanoparticles on the mechanical properties of dental stone. J. Prosthet. Dent., 2012: 1-5.

[10] K.S. Wilson, J.M. Antonucci, Interphase structure property relationships in thermoset dimethacrylate nanocomposites. Dental materials, 2006, 22: 995-1001.

[11] M. Chen, Update on dental nanocomposites. J Dent Res, 2010, 89(6): 549-560.

[12] A. Nanoparticles, Compressive and diametral tensile strength of dental stones with $\mathrm{SiO}_{2}$ and $\mathrm{Al}_{2} \mathrm{O}_{3}$ nanoparticles. IJMSIR, 2018, 3(5).

[13] M.M. Gad, R. Abualsaud, Behavior of PMMA denture base materials containing titanium dioxide nanoparticles: A literature review. International Journal of Biomaterials, 2019.

[14] W. Wang, S. Liao, Y. Zhu, et al., Recent applications of nanomaterials in prosthodontics. Journal of Nanomaterials, 2015, 2015.

[15] H.A. Rodríguez-Quirós, H.F. Casanova-Yepes, Effect of the functionalization of silica nanoparticles as a reinforcing agent on dental composite materials. Rev. Fac. Ing. Univ. Antioquia N. 75, 2015: 36-44.

[16] C. Akay, E. Avukat, Effect of nanoparticle addition on polymethy lmethacrylate resins. Acta Scientific Dental Sciences, 2019, 3(7): 91-97.

[17] P. Fernandes, N. Vassilakos, Accuracy, detail reproduction, and hardness of gypsum casts produced from silicone impressions treated with glow discharge. J Prosthetic Dentistry, 1993, 70(5): 457-464.

[18] I. Vozza, M. Suraci, F. Di Carlo, et al., Dimensional stability of a silica reinforced polyurethane for casts. International Association for Dental Research, 13, Hawaii Convention Center, Hawaii, 2004.

Copyright $($ Ola Mohammed Aljubori, Ali Mohammad Ali Aljafery, and Raja>a Mahdi Al-Mussawi. This is an open-access article distributed under the terms of the Creative Commons Attribution License, which permits unrestricted use, distribution, and reproduction in any medium, provided the original author and source are credited. 